\title{
Simulação baseada em Dinâmica de Sistemas para o ensino da fisiologia do eixo Hipotálamo-hipófise-tireoide no contexto da graduação em medicina
}

\author{
André J. A. Ramos ${ }^{1,2}$, Rafael Oliveira Chaves ${ }^{3}$, Elói Luiz Favero ${ }^{1,2}$ \\ ${ }^{1}$ Universidade Federal do Pará (UFPA) - Instituto de Ciências Exatas e Naturais. Rua \\ Augusto Corrêa, 01 - CEP 66075-110. Caixa postal 479 - Belém - PA - Brasil \\ ${ }^{2}$ Programa de Pós-graduação em Ciência da Computação - PPGCC - UFPA \\ ${ }^{3}$ Universidade do Estado do Pará (UEPA) - Programa de Pós-Graduação Mestrado \\ Profissional em Cirurgia e Pesquisa Experimental - CIPE. \\ Travessa Perebebuí 2623 - CEP: 66.095.661. Belém - PA - Brasil \\ \{andre.rammos 7, rafael.ufpa, favero.ctic\} @gmail.com
}

\begin{abstract}
The use of simulators in medical education has been growing steadily as an inexpensive and effective technology for training. In this work we propose a simulator, for endrocrinology, for teaching the hypothalamicpituitary-thyroid axis (HPT), called EndoSim-LZ which executes a System Dynamics (SD) model for this axis. With it, visually, the student can control the values of any hormone of the axis and verify the repercussion in the production of the others. An assessment was performed to capture the perception of experts through a 14-item Likert scale survey, where some negative points were raised regarding interface and interaction improvements, but the main positive points related to relevance and usefulness in teaching the HPT axis were also raised.
\end{abstract}

Resumo. $O$ uso de simuladores no ensino da medicina, vem crescendo continuamente, como uma tecnologia barata e efetiva para treinamentos. Neste trabalho propomos um simulador, para endrocrinologia, para ensino do eixo hipotálamo-hipófise-tireoide (HHT), denominado EndoSim-LZ que executa um modelo de Dinâmica do Sistema (DS) para esse eixo. Com ele, visualmente, o aluno pode controlar os valores de qualquer hormônio do eixo e verificar a repercussão na produção dos demais. Foi realizada uma avaliação para capturar a percepção de especialistas por meio de um survey com 14 itens na escala Likert, onde foram levantados alguns pontos negativos relacionados à melhorias de interface e interação, mas também foram levantados os principais pontos positivos que estão relacionados à relevância e utilidade no ensino do eixo HHT.

\section{Introdução}

O uso de simuladores na saúde vem crescendo e sendo empregado como metodologia inovadora para treinamento e ensino, pois podem reproduzir a realidade com resultados cada vez mais acurados [Harder 2010]. Por meio das diretrizes educacionais para educação e formação dos profissionais de saúde, a Organização Mundial de Saúde 
VIII Congresso Brasileiro de Informática na Educação (CBIE 2019)

Anais do XXX Simpósio Brasileiro de Informática na Educação (SBIE 2019)

(OMS) recomenda fortemente o emprego de simulação nos cursos de saúde [Tawalbeh and Tubaishat 2013]. Bem como enfatiza sua utilidade, ampla aplicação em diferentes áreas, capacidade de conferir habilidades, satisfação e aceleração do aprendizado do estudante. Os trabalhos de Alfes (2010), Cant and Cooper (2009) concordam que, dentre as habilidades adquiridas pelos estudantes que aprenderam com simulação, as mais percebidas são: o desenvolvimento do raciocínio crítico, o incremento no conhecimento, a confiança própria e a satisfação quando comparadas a métodos de ensino tradicionais.

A simulação é empregada em diferentes disciplinas e áreas da saúde sob vários tipos de metodologias, desde: a encenação de situações clínicas tendo estudantes como atores [Varga et al. 2009]; a utilização de manequins computadorizados que simulam um paciente humano como o SimMan $3 \mathrm{G}^{1}$; modelos computacionais em 3D associados à realidade virtual para o ensino da anatomia, treinamento clínico e cirúrgico [Yoshida et al. 2017]; e réplicas anatômicas reproduzidas com impressão 3D [Park et al. 2018].

A simulação é uma opção inovadora para o ensino de disciplinas complexas que causam menor interesse nos alunos, como é o caso da fisiologia endocrinológica [LellisSantos et al. 2011]. A fisiologia endocrinológica, também composta por seus eixos (e.g. Hipotálamo-hipófise-tireoide, Hipotálamo-hipófise-adrenal, Hipotálamo-hipófisegonadal, eixo somatotrófico, etc), normalmente, não é uma disciplina de preferência de muitos estudantes de medicina por exigir domínio de bioquímica, biologia celular, fisiologia geral e capacidade de relacionar todo esse conhecimento sob uma perspectiva sistêmica [Lellis-Santos et al. 2011, Michael 2007].

Para a modelagem e simulação computacional dos eixos endocrinológicos com finalidade educacional, a Dinâmica de Sistemas (DS) [Pruyt 2013] já é usada como metodologia bastante promissora, uma vez que modelos representando o eixo Hipotálamo-hipófise-tireoide (HHT) já foram realizados por Degon et al. (2008), Dietrich et al. (2012), Seker and Barlas (2012), Han et al. (2016) e Hoermann et al. (2017).

O eixo HHT é composto pelas seguintes glândulas, na ordem crânio caudal: hipotálamo, hipófise e tireóide. O hipotálamo libera o hormônio TRH que estimula a secreção do TSH pela hipófise e o TSH que estimula a liberação de T3 e T4 pela tireoide. Os hormônios produzidos pela tireoide (T3 e T4), dentre outras funções, controlam o metabolismo celular de modo que afetam a fisiologia de quase todos os órgãos e tecidos do corpo [Melmed et al. 2010]. Altos níveis de T3 e T4 no sangue sinalizam a inibição de TRH e TSH, enquanto uma queda na circulação de T3 e T4 estimulará a produção de TRH e TSH.

O eixo HHT também apresenta uma estrutura que dita o comportamento regulatório da produção/inibição de diversos hormônios essenciais no controle das funções e dos processos de órgãos específicos do corpo [Melmed et al. 2010]. Sendo assim, os futuros profissionais da saúde precisam estar capacitados a reconhecer pacientes com distúrbios relacionados a esse eixo endocrinológico. Para isto, devem conhecer e compreender a fisiologia a fim de saber quando existem possíveis anormalidades. Desse modo, espera-se que o emprego da simulação no ensino da

\footnotetext{
${ }^{1}$ Disponível em https://www.laerdal.com/br/products/simulation-training/emergency-care-trauma/simman3g. Acesso em 24 de Junho de 2019.
} 
VIII Congresso Brasileiro de Informática na Educação (CBIE 2019)

Anais do XXX Simpósio Brasileiro de Informática na Educação (SBIE 2019)

fisiologia endocrinológica possa trazer melhorias no conhecimento dos acadêmicos de medicina em relação ao eixo HHT.

Todavia, simuladores como ferramentas para fins didáticos necessitam ser avaliados em relação a sua eficácia de ensino, de forma que seja justificado sua incorporação nas metodologias de ensino [Harder 2010]. As revisões sistemática da literatura realizadas por Harder (2010) e Cant and Cooper (2009), sob diferentes critérios, encontraram trabalhos que, majoritariamente, confirmam a eficácia da simulação, inclusive relatando ganhos adicionais de habilidades, quando comparados aos métodos tradicionais.

Este artigo está organizado da seguinte forma: Seção 2 apresenta a principal proposta do trabalho. Seção 3 apresenta o método de modelagem e simulação utilizado por trás do software, a Dinâmica de Sistema. Seção 4 apresenta alguns trabalhos relacionados, referindo ao uso de simuladores no ensino da endocrinologia. A seção 5 apresenta o modelo DS desenvolvido. A Seção 6, por sua vez, demonstra alguns detalhes da arquitetura, interface e funcionamento do simulador. Já a Seção 7 apresenta resultados e discursão. Finalmente, na seção 8 apresenta-se a conclusão.

\section{Proposta}

Neste trabalho foi desenvolvido um simulador baseado em software, o EndoSim-LZ, para o ensino do eixo endocrinológico HHT, no contexto da graduação em medicina. $\mathrm{O}$ método de simulação DS é usado para descrever, matematicamente, o comportamento fisiológico do eixo, assim representando: a estrutura anatômica, que é formada pelas glândulas hipotálamo, hipófise e tireoide; e as suas relações de feedback positivo e negativo na produção dos hormônios thyrotropin-releasing hormone (TRH), thyroidstimulating hormone (TSH), triodotironina (T3) e tiroxina (T4).

Por meio da interface gráfica do simulador, o acadêmico de medicina poderá controlar a produção dos hormônios TRH, TSH, T3 e T4. Bem como, visualizar graficamente as interações que esses hormônios possuem entre si, causando estímulo/inibição na quantidade produzida de cada um deles. Por exemplo, quando o aluno controla o aumento da produção de TRH, logo haverá o aumento da produção de TSH e, consequentemente, de T3 e T4. De outra forma, se o aluno diminuir a produção de T4, haverá aumento na produção de TRH e de TSH. Assim, o estudante de medicina poderá experimentar um ambiente simulado do comportamento fisiológico desse eixo endocrinológico.

O EndoSim-LZ não tem o objetivo de realizar uma simulação preditiva da quantidade sérica exata dos níveis hormonais. O objetivo se concentra em demonstrar como os hormônios interagem fisiologicamente entre si na produção de cada um, em observância dos feedbacks positivos/negativos. Este trabalho busca melhorias na educação médica, com intuito de capacitar os futuros profissionais da saúde a desenvolver habilidades clínicas por meio do emprego de simulação baseada em DS.

Para uma compreensão preeliminar sobre o potencial uso do EndoSim-LZ como ferramenta de ensino do eixo HHT, foi realizada uma avaliação com especialistas em endocrinologia, por meio de um survey adaptado de Savi et al. (2010). Espera-se que os 
VIII Congresso Brasileiro de Informática na Educação (CBIE 2019)

Anais do XXX Simpósio Brasileiro de Informática na Educação (SBIE 2019)

achados positivos deste trabalho, contribuam no avanço das pesquisas relacionadas ao uso de DS no desenvolvimento de novos simuladores educacionais em saúde.

\section{Dinâmica de Sistemas}

DS é uma metodologia de modelagem e simulação auxiliada por computador, que coloca em prática o pensamento sistêmico e busca explicações endógenas para o comportamento de um sistema qualquer, sendo assim, é capaz de descrever o fucionamento de sistemas fisiológicos dos organismos vivos [Richardson 2014]. Na DS, o foco é estudar o comportamento dos sistemas a partir das causas geradas pelos seus componentes internos [Pruyt 2013]. A DS mapeia as relações causais dos componentes de um sistema através de equações diferenciais interligadas, as quais são representadas por diagramas simples que estruturam o modelo [Pruyt 2013].

Um modelo DS representa as estruturas de estoques e de fluxos dos sistemas, assim como as relações de loops e feedback [Sterman 2001]. A cada uma dessas estruturas são atribuídas funções matemáticas que possibilitam a reprodução do comportamento do sistema [Pruyt 2013]. Uma vez que um sistema é modelado e validado, os feedbacks do comportamento ao longo do tempo de simulação podem ser visualizados em diversos tipos de gráficos ou tabelas [Pruyt 2013]. Um fator bastante proveitoso da flexibilidade e apresentação dos feedbacks é que modelos de DS podem ser convertidos em simuladores e jogos para treinamento, ensino e pesquisa, o que já tem sido feito e obtido bons resultados [Meadows 2007, Barlas and Diker 2000].

Para Rubin et al. (2010), é importante o ensino de DS durante a formação dos médicos para compreensão do comportamento sistêmico do corpo humano em resposta a tratamentos médicos e farmacológicos. Essa abordagem já vem sendo realizada na Escola de Saúde Pública Andrija Stampar, em Zagreb, Croácia, por quase 40 anos nos cursos de saúde, onde métodos de modelagem e simulação com ênfase em DS são ensinados a graduandos e pós-graduandos de medicina [Bozikov et al. 2018].

\section{O uso de simuladores no ensino da endocrinologia}

Tendo o objetivo de encontrar trabalhos relacionados ao uso de modelos de simulação no ensino da endocrinologia, em junho de 2019 foi realizada uma busca nas bases PubMed ${ }^{\circledR}$ e Cochrane Library ${ }^{\circledR}$ usando a seguinte string de busca nos campos título e resumo: (education[Title/Abstract] OR teaching[Title/Abstract] OR learning[Title/Abstract]) AND (endocrinology[Title/Abstract] AND Simulation[Title/Abstract]). A busca retornou sete trabalhos, dos quais foram analisados apenas os artigos (e suas referências) que tratavam do ensino do sistema endocrinológico por meio de simulação computacional.

Os achados mostram modelos computacionais que simulam a estrutura e a fisiologia do eixo HHT, desde os anos 70 e se estende até a década atual [Bergman 1977, Degon et al. 2008, Dietrich et al. 2012, Hoermann et al. 2017]. Atualmente, de acordo com Han et al. (2016), os trabalhos realizados por Eisenberg and DiStefano (apud Han et al., 2016) resultaram na construção de um modelo matemático complexo do eixo HHT e suas relações que demonstrou ser capaz de simular com precisão diversos dados de estudos de caso clínicos. O mesmo autor também afirma que, embora 
VIII Congresso Brasileiro de Informática na Educação (CBIE 2019)

Anais do XXX Simpósio Brasileiro de Informática na Educação (SBIE 2019)

tal modelo tenha tamanha aplicação e precisão, ele ainda não tem sido muito explorado em razão de sua complexidade matemática.

No mesmo artigo, Han et al. (2016) avalia a precisão de uma aplicação Web, denominada THYROSIM ${ }^{2}$, que executa simulações baseadas no modelo de Eisenberg and DiStefano. No entanto, apesar de sua aplicabilidade educacional também ser enfatizada, tal trabalho se propôs a demonstrar apenas os efeitos fisiológicos do uso inadequado de hormônios tireoidianos (TH's) vendidos sem receita médica.

O simulador SimThyr ${ }^{3}$ tem como objetivo apoiar pesquisas e a educação em tireoidologia [Dietrich 2016]. As pesquisas que utilizaram o software contribuíram significativamente para indiciar e provar diversas hipóteses dentro da área, além de já ser empregado também na educação médica [Dietrich et al. 2016, Hoermann et al. 2015, Dietrich et al. 2012].

Na System Dynamics Review ${ }^{4}$ também foi encontrado o trabalho de Seker and Barlas (2012). Este foi o modelo do eixo HHT mais abrangente, desenvolvido com DS, encontrado na literatura e se propôs a modelar o sistema hormonal da tireoide para retratar a dinâmica dos hormônios principais em estados mórbidos e saudáveis, enfatizando o papel dos mecanismos de feedback funcional existente no sistema. $\mathrm{O}$ modelo construído foi validado analisando os aspectos estruturais e comportamentais. Também foi percebido, nos achados da busca, que nenhum utiliza simulação de DS exclusivamente para ensino da endocrinologia por meio de simulação computacional, que é o foco deste trabalho.

\section{O modelo}

O modelo DS do eixo HHT do simulador foi desenvolvido no software Vensim (ver Figura 1), a partir das equações do modelo matemático simplificado por Martins and Monteiro (2010). No Vensim foi possível plotar os dados resultantes da reprodução do modelo e visualizá-los em gráfico, que posteriormente foram avaliados de acordo com o comportamento esperado da variação de cada hormônio em relação ao outro. Os estoques foram configurados para iniciarem com os valores de equilíbrio definidos em Martins and Monteiro (2010) e é assumido serem estes os valores normais do indivíduo saudável.

\footnotetext{
${ }^{2}$ Disponível em http://biocyb1.cs.ucla.edu/thyrosim/. Acesso 24 de junho de 2019.

${ }^{3}$ Disponível em https://simthyr.sourceforge.io/. Acesso 28 de Junho de 2019.

${ }^{4}$ Disponível em https://www.systemdynamics.org/. Acesso 26 de junho de 2019.
} 
VIII Congresso Brasileiro de Informática na Educação (CBIE 2019)

Anais do XXX Simpósio Brasileiro de Informática na Educação (SBIE 2019)

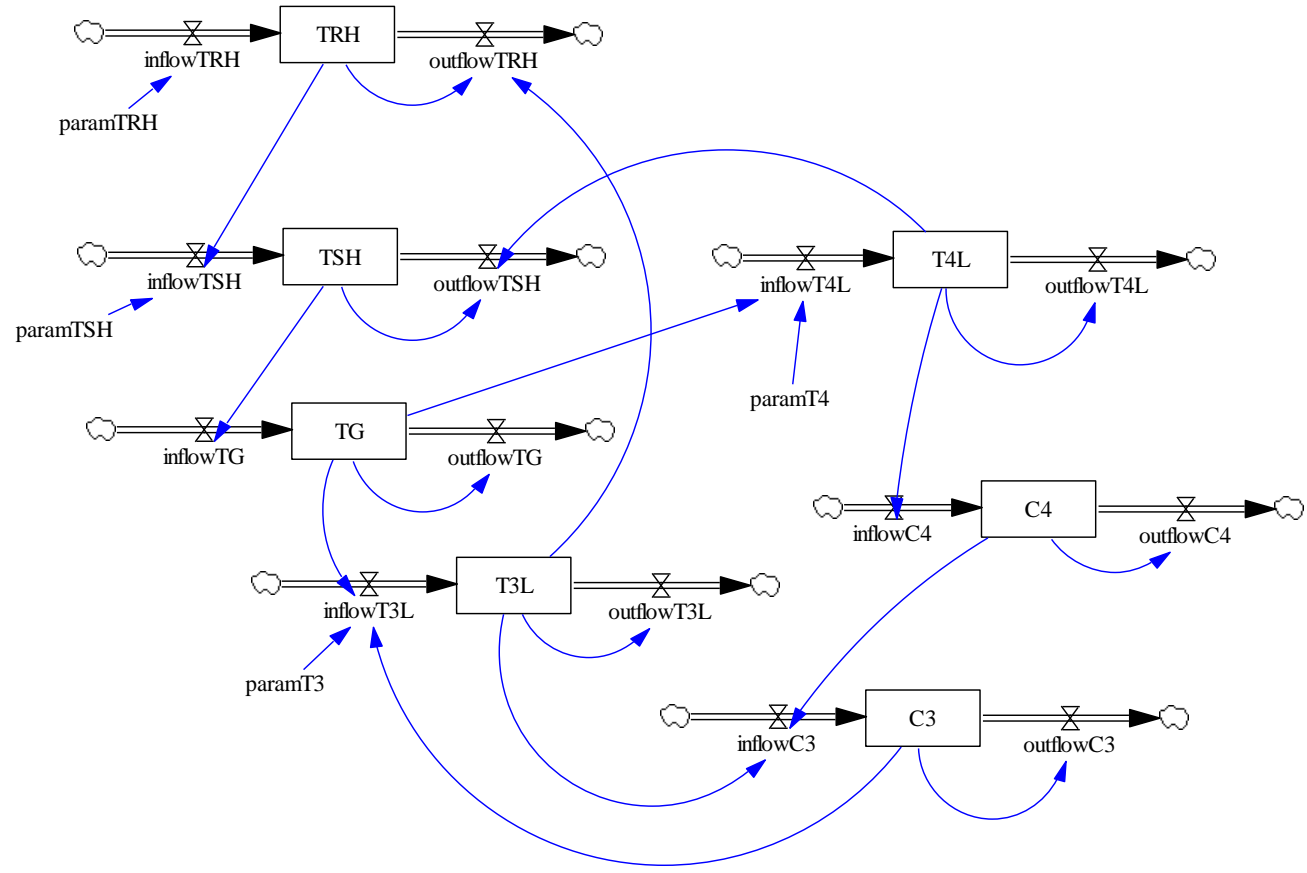

Figura 1. Modelo DS construído com o software VenSim a partir das equações do trabalho de [Martins and Monteiro 2010].

\section{O simulador}

O simulador EndoSim-LZ foi desenvolvido na linguagem Python 2.7. Para a interface gráfica foi utilizada a biblioteca open source Kivy. ${ }^{5}$ A biblioteca PySD ${ }^{6}$ é usada para transformar e manipular os dados do modelo DS do eixo HHT, que foi criado no software de modelagem de DS VenSim ${ }^{7}$. Conforme descrito na arquitetura da Figura 2, o software Vensim gera o arquivo modelo.mdl que, por intermédio da biblioteca PySD, é transformado em um arquivo modelo.py contendo funções e atributos que tornam os dados da simulação manipuláveis. $\mathrm{O}$ arquivo main.py é o arquivo principal que cria a interface gráfica por meio dos recursos da biblioteca Kivy e manipula os dados da simulação por meio de funções da biblioteca PySD.

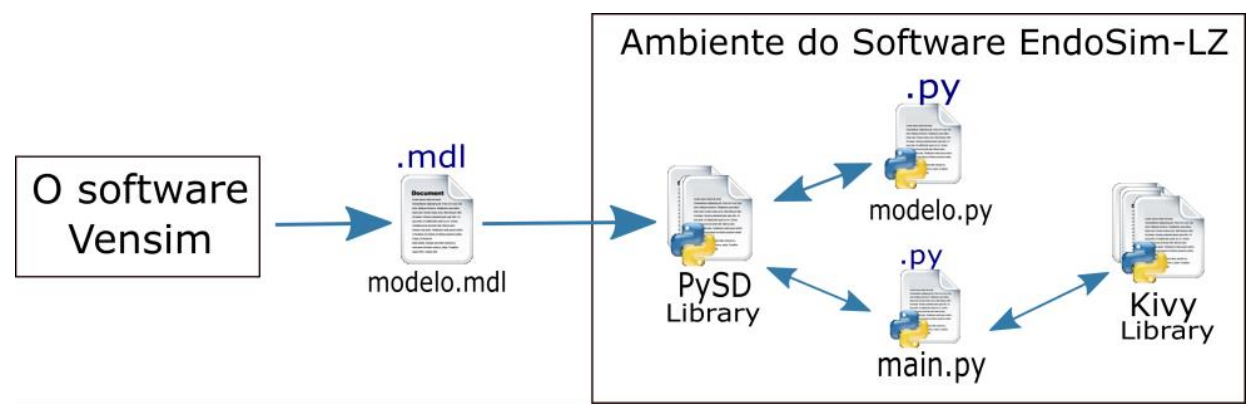

Figura 2. Arquitetura do sistema. As setas duplas indicam a possibilidade de um arquivo ou biblioteca interagir um com outro, sendo o main.py 0 arquivo principal da aplicação.

\footnotetext{
${ }^{5}$ Disponível em https://kivy.org/. Acessado em 26 de junho de 2019.

${ }^{6}$ Disponível em https://pysd.readthedocs.io/en/master/. Acessado em 30 de junho de 2019.

${ }^{7}$ Disponível em https://vensim.com/vensim-software/. Acessado em 26 de junho de 2019.
} 
VIII Congresso Brasileiro de Informática na Educação (CBIE 2019)

Anais do XXX Simpósio Brasileiro de Informática na Educação (SBIE 2019)

O EndoSim-LZ foi desenvolvido para ser executado em dispositivos do tipo desktop, em que o usuário poderá interagir e visualizar os resultados simulados na produção dos hormônios. A Figura 3 representa a tela principal do simulador, e as funcionalidades são:

i. Controle da produção hormonal: A produção individual de cada um dos 4 hormônios pode ser controlados por botões verticais de aumentar/diminuir;

ii. Visualização da quantidade da produção hormonal de cada glândula: Em uma imagem 3D do eixo hipotálamo-hipofisário-tireoide humano (produzida pelo projeto) círculos representam a quantidade de hormônios produzida. O círculo azul representa a quantidade de TRH, o verde de TSH, o laranja T3 e o vermelho de T4. Os círculos variam de diâmetro, indicando a produção/inibição de cada de acordo com a relação de feedback negativos.

iii. Gráfico de linhas: representa a relação entre as produções hormonais no tempo.

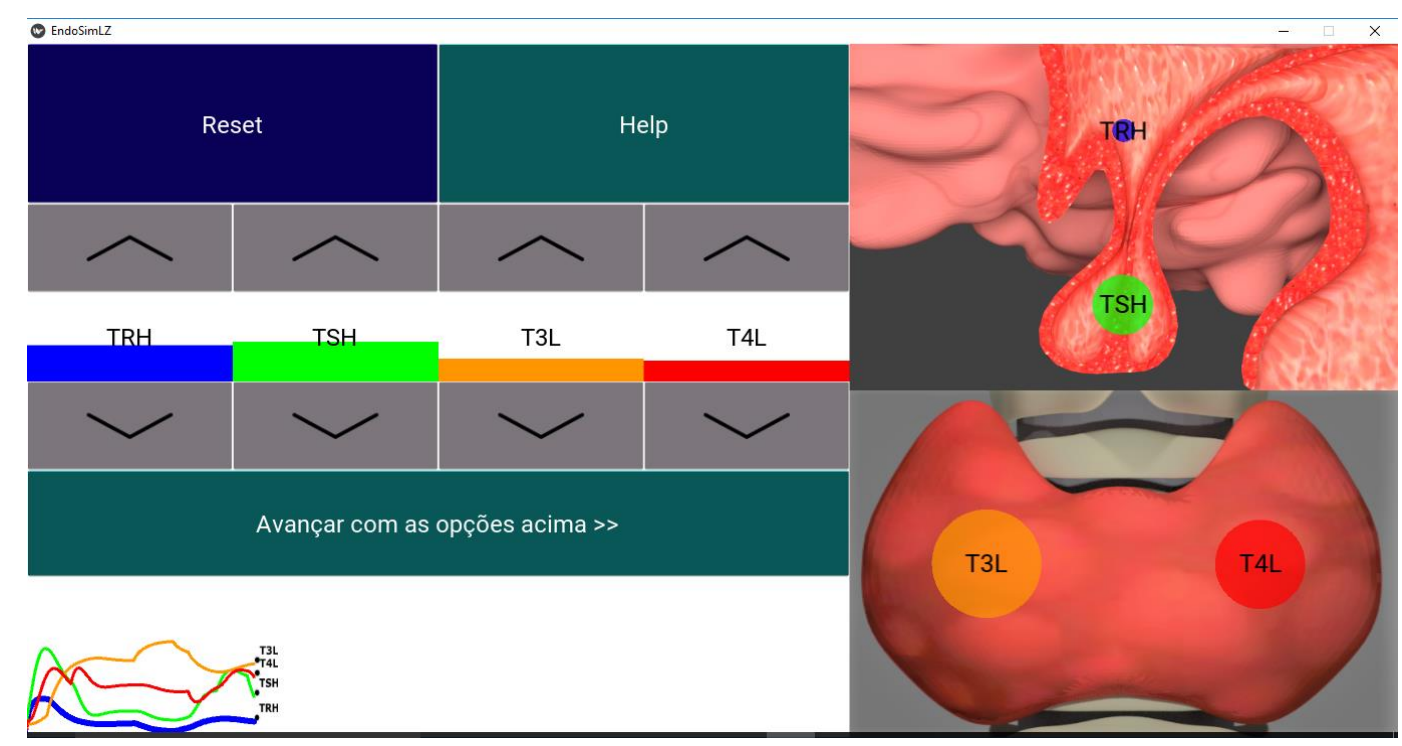

Figura 3. (i) A produção hormonal controlada por botões de aumentar/diminuir; (ii) representação das glândulas envolvidas no eixo HHT, com círculos representando as relações de feedback positivo/negativo; (iii) gráfico de linhas evidenciando relações de feedback.

$\mathrm{Na}$ tela também existe um botão de ajuda ao usuário e outro para resetar os valores, como mostra a Figura 3.

\section{Resultados e discussão}

O simulador foi avaliado por 3 especialistas em endocrinologia do Centro Hospitalar Jean Bitar em Belém do Pará, Brasil. Nesta avaliação foi utilizado um survey adaptado sobre a eficácia educacional de jogos, uma vez que, as simulações são um tipo de jogo educativo [Savi et al. 2010]. Para isso, alguns dos itens originais de Savi et al. (2010) foram abstraídos ou modificados, utilizando apenas aqueles que tinham maior relevância para avaliar um simulador. No survey, os avaliadores atribuiram uma nota na escala de Likert indicando o seu grau de concordância para cada item objetivo, sendo: -2 para "discordo fortemente", -1 para "discordo", 0 para "neutro", 1 para "concordo" e 2 para "concordo fortemente". 
VIII Congresso Brasileiro de Informática na Educação (CBIE 2019)

Anais do XXX Simpósio Brasileiro de Informática na Educação (SBIE 2019)

Observando os itens avaliados e suas respectivas médias (ver Figura 4), percebese que as questões que ganharam um baixo nível de interesse de alguns avaliadores foram as relacionadas ao uso (item 6) e ao interesse em aprender mais (item 3). Isso deve-se ao fato de eles serem especialistas na área e já terem estudado bastante o assunto, sendo esperado que o simulador não tenha proporcionado aos mesmos uma compreensão a mais sobre o eixo (item 12 e 13). É importante observar que os itens 7 , $8,9,10$ e 11, que tratam da dificuldade de uso, excesso de conteúdo e entedimento do simulador foram baixos.

Os itens mais bem avaliados (item 2,5 e 14) estavam relacionados à clareza com que o simulador conseguiu reproduzir e transmitir o conhecimento sobre o eixo ao usuário. Em suma, os principais pontos negativos estão relacionados à interface (item 1) e interação, os principais pontos positivos estão relacionados à relevância, utilidade e interesse em aprender mais sobre o eixo HHT. O design da interface, embora tenha sido relativamente simples, foi desenvolvido levando em consideração as normas da ISO 9241 em Medeiros (1999) que se referem a clareza, concisão, discriminação, consistência, legibilidade e compreensibilidade das informações na tela.

\begin{tabular}{|c|c|c|c|}
\hline ITEM AVALIADO & MÉDIA & ITEM AVALIADO & MÉDIA \\
\hline $\begin{array}{l}\text { 1- O design da interface do Simulador é } \\
\text { atraente? }\end{array}$ & & $\begin{array}{l}\text { 8- O Simulador tinha tanta informação que foi } \\
\text { difícil identificar e lembrar dos pontos }\end{array}$ & \\
\hline $\begin{array}{l}\text { 2- Ficou claro para mim como o conteúdo do } \\
\text { Simulador está relacionado com coisas que eu já } \\
\text { sabia sobre o sistema endócrino. }\end{array}$ & & $\begin{array}{l}\text { 9- O conteúdo do Simulador é tão abstrato que } \\
\text { foi difícil manter a atenção nele. }\end{array}$ & \\
\hline $\begin{array}{l}\text { 3- Eu gostei tanto do Simulador que gostaria de } \\
\text { aprender ainda mais sobre o assunto abordado }\end{array}$ & & $\begin{array}{l}\text { 10- As atividades do Simulador foram muito } \\
\text { difíceis. }\end{array}$ & \\
\hline $\begin{array}{l}\text { 4- O conteúdo do Simulador é bastante relevante } \\
\text { para meus interesses. }\end{array}$ & & $\begin{array}{l}\text { 11- Eu não consegui entender uma boa parcela } \\
\text { do conteúdo e comportamento exposto na }\end{array}$ & \\
\hline $\begin{array}{l}\text { 5- Eu poderia relacionar o conteúdo do } \\
\text { Simulador com coisas que já vi, fiz ou pensei. }\end{array}$ & & $\begin{array}{l}\text { 12- Depois do Simulador consigo lembrar de } \\
\text { mais informações relacionadas a endocrinologia } \\
\text { apresentados por ele. }\end{array}$ & 0 , \\
\hline $\begin{array}{l}\text { 6- O poder didático do Simulador será útil para } \\
\text { mim. }\end{array}$ & & $\begin{array}{l}\text { 13- Depois do Simulador consigo compreender } \\
\text { melhor sobre o comportamento do sistema }\end{array}$ & \\
\hline $\begin{array}{l}\text { 7- O Simulador foi mais difícil de entender do } \\
\text { que eu gostaria. }\end{array}$ & & $\begin{array}{l}\text { 14- Depois de usar o Simulador sinto que } \\
\text { consigo aplicar melhor o que aprendi. }\end{array}$ & \\
\hline
\end{tabular}

Figura 4. Itens avaliados e suas respectivas médias.

\section{Conclusão}

Trabalhos com simulação na área da educação médica já foram feitos para diversos temas e vem sendo crescentemente incentivados pela OMS. A simulação matemática auxiliada por softwares tem sido utilizada com mais frequência em experimentos preditivos, protelando sua utilidade educacional. A DS também tem sido uma proposta promissora para o ensino e modelagem de sistemas que contenham estoque e fluxos dos quais os sistemas fisiológicos fazem parte. Todavia, é relevante dizer que faltam trabalhos que explorem ou investiguem esse potencial na educação médica. Neste trabalho foi desenvolvido um simulador para ensino do eixo HHT que executa um modelo de DS. A partir da boa aceitação dos especialistas bem como das críticas, estamos planejando os trabalhos futuros, que consistem em:

i. Aplicar e avaliar experimentalmente o simulador com grupos controle e pré/pós teste;

ii. Desenvolver a versão web do simulador para facilitar sua portabilidade; 
VIII Congresso Brasileiro de Informática na Educação (CBIE 2019)

Anais do XXX Simpósio Brasileiro de Informática na Educação (SBIE 2019)

iii. Desenvolver uma interface de programação de aplicação (API) que também facilite a transformação de modelos DS construídos com o software Vensim para a linguagem C-sharp, facilitando o desenvolvimento de outros aplicativos para educação médica com a Game Engine do Unity 3D associados à metodologia DS.

É importante enfatizar que, embora poucos especialistas tenham sido consultados, percebe-se a existência de bons indícios de viabilidade como software educacional para o simulador, sendo que, numa versão mais ampla do estudo, desenvolveremos uma avaliação que apresente um maior nível de evidência do impacto da ferramenta na aprendizagem. Isto quando for realizada a avaliação experimental com pré/pós teste e com grupos controle. Assim, espera-se que os achados positivos deste trabalho, contribuam no avanço das pesquisas relacionadas ao uso de DS no desenvolvimento de novos simuladores educacionais em saúde.

\section{Referências}

Alfes, C. M. (2010). Evaluating the use of simulation with beginning nursing students. Journal of Nursing Education, 50(2):89-93.

Barlas, Y. and Diker, V. G. (2000). A dynamic simulation game (UNIGAME) for strategic university management. Simulation \& Gaming,31(3):331-358.

Bozikov, J., Relic, D., and Dezelic, G. (2018). Use of system dynamics modeling in medical education and research projects. Studies in health technology and informatics, 247:830-834.

Cant, R. P. and Cooper, S. J. (2009). Simulation-based learning in nurse education: systematic review. Journal of Advanced Nursing, 66(1):3-15.

Degon, M., Chipkin, S. R., Hollot, C., Zoeller, R. T., and Chait, Y. (2008). A computational model of the human thyroid. Mathematical Biosciences.

Dietrich, J. W., Landgrafe, G., and Fotiadou, E. H. (2012). TSH and thyrotropic agonists: Key actors in thyroid homeostasis. Journal of Thyroid Research, 2012.

Dietrich, J. W., Landgrafe-Mende, G., Wiora, E., Chatzitomaris, A., Klein, H. H., Midgley, J. E. M., and Hoermann, R. (2016). Calculated parameters of thyroid homeostasis: Emerging tools for differential diagnosis and clinical research. Frontiers in Endocrinology, 7.

Han, S. X., Eisenberg, M., Larsen, P. R., and DiStefano, J. (2016). THYROSIM app for education and research predicts potential health risks of over-the-counter thyroid supplements. Thyroid,26(4):489-498.

Harder, B. N. (2010). Use of simulation in teaching and learning in health sciences: A systematic review. Journal of Nursing Education, 49(1):23-28.

Hoermann, R., Midgley, J. E. M., Larisch, R., and Dietrich, J. W. (2015). Homeostatic control of the thyroid-pituitary axis: Perspectives for diagnosis and treatment. Frontiers in Endocrinology, 6.

Hoermann, R., Midgley, J. E. M., Larisch, R., and Dietrich, J. W. C. (2017). Advances in applied homeostatic modelling of the relationship between thyrotropin and free thyroxine. PLOS ONE, 12(11):e0187232. 
VIII Congresso Brasileiro de Informática na Educação (CBIE 2019)

Anais do XXX Simpósio Brasileiro de Informática na Educação (SBIE 2019)

Lellis-Santos, C., Giannocco, G., and Nunes, M. T. (2011). The case of thyroid hormones: how to learn physiology by solving a detective case. Advances in Physiology Education, 35(2):219-226.

Martins, L. G. and Monteiro, L. H. (2010). A model of intelligent controller for hypothyroidism treatment. In 2010 10th IEEE International Conference on Computer and Information Technology. IEEE.

Meadows, D. (2007). A brief and incomplete history of operational gaming in system dynamics. System Dynamics Review, 23(2-3):199-203.

Medeiros, M. A. (1999) ISO 9241: uma proposta de utilização da norma para avaliação do grau de satisfação de usuários de software Universidade Federal de Santa Catarina. MastersThesis.

Melmed, S., Polonsky, K., Larsen, P. R., and Kronenberg, H. (2010). Williams Tratado de Endocrinologia (Em Portuguese do Brasil). Elsevier Professional.

Michael, J. (2007). What makes physiology hard for students to learn? results of a faculty survey. Advances in Physiology Education, 31(1):34-40.

Park, H. J., Wang, C., Choi, K. H., and Kim, H. N. (2018). Use of a life-size threedimensional-printed spine model for pedicle screw instrumentation training. Journal of Orthopaedic Surgery and Research, 13(1).

Pruyt, E. (2013). Small System Dynamics Models for Big Issues: Triple Jump towards Real-World Complexity. TU Delft Library, Delft, The Netherlands, 1.0 edition.

Richardson, G. P. (2014). "model” teaching II: Examples for the early stages. System Dynamics Review, 30(4):283-290.

Rubin, D. M., Richards, C. L., Keene, P. A. C., Paiker, J. E., Gray, A. R. T., Herron, R. F. R., Russell, M. J., and Wigdorowitz, B. (2010). System dynamics in medical education: a tool for life. Advances in Health Sciences Education, 17(2):203-210.

Savi, R., Wangenheim, C. G. V., Ulbricht, V., and Vanzin, T. (2010). Proposta de um modelo de avaliação de jogos educacionais. RENOTE, 8(3).

Seker, O.; Barlas, Y. . A. F. ((2012)). Modelling the dynamics of thyroid hormones and related disorders. Master's thesis, Bogazici University.

Sterman, J. D. (2001). System dynamics modeling: Tools for learning in a complex world. California Management Review, 43(4):8-25.

Tawalbeh, L. I. and Tubaishat, A. (2013). Effect of simulation on knowledge of advanced cardiac life support, knowledge retention, and confidence of nursing students in jordan. Journal of Nursing Education.

Varga, C. R. R., de Carvalho Almeida, V., Germano, C. M. R., Melo, D. G., Chachá, G. F., Souto, B. G. A., Fontanella, B. J. B., and Lima, V. V. (2009). Relato de experiência: o uso de simulações no processo de ensino-aprendizagem em medicina. Revista Brasileira de Educação Médica.

Yoshida, E. A., Castro, M. L. A., and Martins, V. F. (2017). Virtual reality and fetal medicine - a systematic review. In 2017 XLIII Latin American Computer Conference (CLEI). IEEE. 